\title{
AN APPROXIMATION TO INFINITELY DIVISIBLE LAWS
}

\author{
S. CSÖRGÖ* (Ann Arbor) \\ Tandori Károly professzor hetvenedik születésnapjára, tanítványi tisztelettel
}

\section{The approximation}

One question Professor Tandori asked at my doctoral defense on February 2,1972 , was about infinite divisibility. Since he was satisfied, my answer probably included that, according to Lévy's formula ([9], p. 84), a distribution on the real line $\mathbf{R}$ is infinitely divisible if and only if its characteristic function $\varphi(t), t \in \mathbf{R}$, is given by

$$
\begin{gathered}
\varphi(t)=\exp \left\{i \theta t-\frac{\sigma^{2}}{2} t^{2}+\int_{-\infty}^{0}\left(e^{i t x}-1-\frac{i t x}{1+x^{2}}\right) d L(x)+\right. \\
\left.+\int_{0}^{\infty}\left(e^{i t x}-1-\frac{i t x}{1+x^{2}}\right) d R(x)\right\}
\end{gathered}
$$

where $i$ is the imaginary unit, $\theta \in \mathbf{R}$ and $\sigma \geqq 0$ are constants, the function $L(\cdot)$ is left-continuous and non-decreasing on $(-\infty, 0)$ with $L(-\infty)=0$ and the function $R(\cdot)$ is right-continuous and non-decreasing on $(0, \infty)$ with $R(\infty)=0$, such that

$$
\int_{-\varepsilon}^{0} x^{2} d L(x)+\int_{0}^{\varepsilon} x^{2} d R(x)<\infty \text { for every } \varepsilon>0
$$

Little did I think at the time that I should be able to answer the question somewhat more thoroughly twenty-three years later. I hope he will like a few late details here.

For a given quadruple $(\theta, \sigma, L(\cdot), R(\cdot))$ with the described properties, let $F_{\theta, \sigma, L, R}(\cdot)$ denote the corresponding distribution function, so that $\varphi(t)=$ 9208067 .

* Partially supported by the National Science Foundation of the U.S.A., Grant DMS- 
$=\int_{-\infty}^{\infty} e^{i t x} d F_{\theta, \sigma, L, R}(x), t \in \mathbf{R}$. Consider the inverse functions

$$
\psi_{L}(u):=\inf \{x<0: L(x)>u\}
$$

and

$$
\psi_{R}(u):=\inf \{x<0:-R(-x)>u\}, 0<u<\infty,
$$

where the infimum of the empty set is taken to be zero. These are nondecreasing, non-positive, right-continuous functions on the half-line $(0, \infty)$ such that

$$
\int_{\varepsilon}^{\infty} \psi_{L}^{2}(u) d u+\int_{\varepsilon}^{\infty} \psi_{R}^{2}(u) d u<\infty \quad \text { for every } \varepsilon>0 .
$$

Let $Y_{1}, Y_{2}, \ldots$ be independent exponentially distributed random variables with mean 1 , so that $P\left\{Y_{k}>x\right\}=e^{-x}, x>0, k \in \mathrm{N}$, and consider the corresponding partial sums $S_{n}:=Y_{1}+\cdots+Y_{n}, n \in \mathbf{N}$. Let $Z$ be a standard normal random variable, let $\left\{S_{n}^{(L)}\right\}_{n=1}^{\infty}$ and $\left\{S_{n}^{(R)}\right\}_{n=1}^{\infty}$ be distributionally equivalent copies of the sequence $\left\{S_{n}\right\}_{n=1}^{\infty}$ such that the sequences $\left\{S_{n}^{(L)}\right\}_{n=1}^{\infty},\left\{S_{n}^{(R)}\right\}_{n=1}^{\infty}$ and $Z$ are independent. Now consider

$$
V_{n}^{(M)}:=\sum_{j=1}^{n} \psi_{M}\left(S_{j}^{(M)}\right)-\int_{1}^{S_{n}^{(M)}} \psi_{M}(u) d u, \quad n \in \mathbf{N}, \quad M=L, R
$$

and the problem of approximating $F_{\theta, \sigma, L, R}(\cdot)$ by the distribution functions $F_{\theta, \sigma, L, R}^{n, m}(x):=P\left\{V_{n}^{(L)}+\sigma Z-V_{m}^{(R)}+\theta-\theta_{L}+\theta_{R} \leqq x\right\}, \quad x \in \mathbf{R}, \quad n, m \in \mathbf{N}$, where

$$
\theta_{M}:=\int_{0}^{1} \frac{\psi_{M}(s)}{1+\psi_{M}^{2}(s)} d s-\int_{1}^{\infty} \frac{\psi_{M}^{3}(s)}{1+\psi_{M}^{2}(s)} d s, \quad M=L, R
$$

More precisely, we are interested in seeing how fast the Lévy distances $D_{n, m}(L, R)$ between $F_{\theta, \sigma, L, R}^{n, m}(\cdot)$ and $F_{\theta, \sigma, L, R}(\cdot)$, defined as

$$
\begin{gathered}
\inf \left\{\varepsilon>0: F_{\theta, \sigma, L, R}^{n, m}(x-\varepsilon)-\varepsilon \leqq F_{\theta, \sigma, L, R}(x) \leqq F_{\theta, \sigma, L, R}^{n, m}(x+\varepsilon)+\varepsilon\right. \\
\text { for all } x \in \mathbf{R}\},
\end{gathered}
$$

go to zero as $n, m \rightarrow \infty$. As it turns out, this depends upon how fast the functions $\psi_{L}(u)$ and $\psi_{R}(u)$ approach zero as $u \rightarrow \infty$. 
For $M=L$ and $M=R$ and any $a>0$ consider

$$
\left\{\begin{array}{l}
v_{M}(a):=\sqrt{\int_{a}^{\infty} \psi_{M}^{2}(u) d u}, \text { so that }\left|\psi_{M}(a)\right| \downarrow 0 \text { and } \\
v_{M}(a) \downarrow 0 \text { as } a \uparrow \infty,
\end{array}\right.
$$

and for a fixed $1 / 2 \leqq d_{M} \leqq 2 e^{(e-2) / 2}$ choose a finite $a_{M}^{*}>0$ so that

$$
\left\{\begin{array}{l}
\psi_{M}\left(a_{M}^{*}\right)=0 \text { if } \psi_{M}(a)=0 \text { for some } a>0 \text { and } \\
v_{M}\left(a_{M}^{*}\right)<\frac{1}{e^{2 / e}} \text { and }\left|\psi_{M}\left(a_{M}^{*}\right)\right| \leqq \frac{1}{e^{e / 2} / d_{M}} \text { if } \psi_{M}(a)<0 \text { for all } a>0,
\end{array}\right.
$$

and, with $\log$ standing for the natural logarithm, for all $a \geqq a_{M}^{*}$ define

$$
:= \begin{cases}w_{1}^{(M)}(a):=\sqrt{\frac{e}{2}} v_{M}(a) \sqrt{\log \frac{1}{v_{M}(a)}}, & \text { if } \sqrt{\frac{2}{e} \log \frac{1}{v_{M}(a)}} \leqq \frac{v_{M}(a)}{\left|\psi_{M}(a)\right|}, \\ w_{2}^{(M)}(a):=\frac{1}{2}\left|\psi_{M}(a)\right| \log \frac{1}{v_{M}(a)\left|\psi_{M}(a)\right|}, & \text { if } \sqrt{\frac{2}{e} \log \frac{1}{v_{M}(a)}}>\frac{v_{M}(a)}{\left|\psi_{M}(a)\right|} \geqq 1, \\ w_{3}^{(M)}(a):=d_{M}\left|\psi_{M}(a)\right| \log \frac{1}{\left|\psi_{M}(a)\right|}, & \text { if } \frac{v_{M}(a)}{\left|\psi_{M}(a)\right|}<1<\sqrt{\frac{2}{e} \log \frac{1}{v_{M}(a)}},\end{cases}
$$

where, since $d_{M} \leqq 2 e^{(e-2) / 2}$, the second inequality in the specification of $w_{3}^{(M)}(a)$ is satisfied because $v_{M}(a)<\left|\psi_{M}(a)\right| \leqq e^{-e^{e / 2} / d_{M}} \leqq e^{-e / 2}$. While it is understood that $w_{M}(a):=0$ if $\psi_{M}(a)=0$, since otherwise $2 w_{2}^{(M)}(a) \leqq$ $\leqq\left|\psi_{M}(a)\right| \log \left(1 /\left|\psi_{M}(a)\right|\right)+v_{M}(a) \log \left(1 / v_{M}(a)\right)$, it is clear that $w_{M}(a) \rightarrow$ $\rightarrow 0$ as $a \rightarrow \infty, M=L, R$. Finally, setting

$$
r_{n}^{(M)}(a):= \begin{cases}P\left\{S_{n} \leqq a\right\}+2 w_{M}(a), & \text { if } \psi_{M}(\cdot) \not \equiv 0 \text { on }(0, \infty), \\ 0, & \text { if } \psi_{M}(\cdot) \equiv 0 \text { on }(0, \infty)\end{cases}
$$

for $M=L, R$ and $a \geqq a_{M}^{*}$, the main result is the following.

THEOREM. If $a_{L} \geqq a_{L}^{*}$ and $a_{R} \geqq a_{R}^{*}$, then $D_{n, m}(L, R) \leqq r_{n}^{(L)}\left(a_{L}\right)+$ $+r_{m}^{(R)}\left(a_{R}\right)$ for every $n, m \in \mathbf{N}$.

It will be also clear from the proof (and will be followed in bracketed phrases) that in the case when $\psi_{M}(u)<0$ for all $u>0$, if $w_{M}(a)=w_{1}^{(M)}(a)$ for all $a \geqq \tilde{a}_{M}$ and $v_{M}\left(\bar{a}_{M}\right) \leqq e^{-2 / e}$ for some $\tilde{a}_{M}, \bar{a}_{M}>0$, then the choice 
$a_{M}^{*}=\max \left(\tilde{a}_{M}, \bar{a}_{M}\right)$ is permissible, while if $w_{M}(a)=w_{2}^{(M)}(a)$ for all $a \geqq \tilde{a}_{M}$ and $\left|\psi_{M}\left(\bar{a}_{M}\right)\right|<1, v_{M}\left(\bar{a}_{M}\right)<1$ and the product $v_{M}\left(\bar{a}_{M}\right)\left|\psi_{M}\left(\bar{a}_{M}\right)\right| \leqq e^{-2}$ for some $\tilde{a}_{M}, \bar{a}_{M}>0$, then again we may take $a_{M}^{*}=\max \left(\tilde{a}_{M}, \bar{a}_{M}\right), M=$ $=L, R$. The constant $d_{M}$ enters the threshold $a_{M}^{*}$ as in (1.5) only if the case $w_{M}(a)=w_{3}^{(M)}(a)$ cannot be excluded for $M=L$ or $M=R$.

To use the theorem, one will choose two positive sequences $\left\{a_{n}^{(L)}: n \in\right.$ $\in \mathbf{N}\}$ and $\left\{a_{n}^{(R)}: n \in \mathbf{N}\right\}$ such that $\lim \sup _{n \rightarrow \infty} a_{n}^{(M)} / n<1, M=L, R$, and obtain $D_{n, m}(L, R) \leqq r_{n}^{(L)}\left(\boldsymbol{a}_{n}^{(L)}\right)+r_{m}^{(R)}\left(\boldsymbol{a}_{m}^{(R)}\right)$ for all $n$ and $m$ such that $\boldsymbol{a}_{n}^{(L)} \geqq$ $\geqq a_{L}^{*}$ and $a_{m}^{(R)} \geqq a_{R}^{*}$. For $a_{n} \equiv a_{n}^{(L)}$ or $a_{n} \equiv a_{n}^{(R)}$, the limsup condition is to force the gamma probabilities

$$
P\left\{S_{n} \leqq a_{n}\right\}=\int_{0}^{a_{n}} \frac{x^{n-1}}{(n-1) !} e^{-x} d x
$$

go to zero as $n \rightarrow \infty$. This convergence is the fastest if $a_{n} \equiv a$ for some $a \geqq$ $a_{L}^{*}$ or $a \geqq a_{R}^{*}$, in which case an expansion of the incomplete gamma function $([8]$, p. 135) yields

$$
P\left\{S_{n} \leqq a\right\}=\frac{a^{n}}{n !} e^{-a} \sum_{k=0}^{\infty} \frac{a^{k}}{(n+1)(n+2) \cdots(n+k)} \leqq \frac{a^{n}}{n !}, \quad n \in \mathbf{N} .
$$

On the other hand, $w_{M}\left(a_{n}\right) \rightarrow 0$ fast for $M=L$ or $M=R$ if $a_{n} \rightarrow \infty$ fast as $n \rightarrow \infty$. For the fastest possible sequence $a_{n} \equiv \tau n$, the elementary Lemma 3.1 in [7] gives

$$
P\left\{S_{n} \leqq \tau n\right\} \leqq e^{-(1-\tau)^{2} n / 2} \quad \text { whenever } \quad 0<\tau<1, \quad n \in \mathbf{N} .
$$

In a concrete situation a trade-off between the opposing tendencies has to be found.

If the limiting infinitely divisible distribution function $F_{\theta, \sigma, L, R}(\cdot)$ is absolutely continuous with density $f_{\theta, \sigma, L, R}(\cdot)$ for which $K_{\theta, \sigma, L, R}:=$ $:=\sup \left\{f_{\theta, \sigma, L, R}(x): x \in \mathbf{R}\right\}<\infty$, then by the theorem and a well-known inequality connecting the Kolmogorov and Lévy distances, for any two positive sequences $\left\{a_{n}^{(L)}: n \in \mathbf{N}\right\}$ and $\left\{a_{n}^{(R)}: n \in \mathbf{N}\right\}$ as above,

$$
\begin{gathered}
\sup _{x \in \mathbf{R}}\left|F_{\theta, \sigma, L, R}^{n, m}(x)-F_{\theta, \sigma, L, R}(x)\right| \leqq \\
\leqq\left[1+K_{\theta, \sigma, L, R}\right]\left[r_{n}^{(L)}\left(a_{n}^{(L)}\right)+r_{m}^{(R)}\left(a_{m}^{(R)}\right)\right]
\end{gathered}
$$

for all $n$ and $m$ such that $a_{n}^{(L)} \geqq a_{L}^{*}$ and $a_{m}^{(R)} \geqq a_{R}^{*}$. 
Another general corollary is for the case when the Lévy measure of the underlying infinitely divisible distribution is finite, i.e. in our terminology, both $L(0-)<\infty$ and $R(0+)>-\infty$. In this case, $\psi_{L}(\cdot)$ is zero on the halfline $\left[L\left(0_{-}\right), \infty\right)$ and $\psi_{R}(\cdot)$ is zero on the half-line $[-R(0+), \infty)$, and the theorem and (1.7) together yield

$$
D_{n, m}(L, R) \leqq \frac{[L(0-)]^{n}}{n !}+\frac{[-R(0+)]^{m}}{m !} \text { for all } n, m \in \mathbf{R} \text {. }
$$

A result of the type of the theorem, though somewhat different in nature, was first proved by Hall [10] for the approximation of stable laws. A closer version was derived among other results in [2]. Stable laws are considered among the illustrative examples in Section 3, following the proof. The theorem above improves the main result in [3], where a special integrability condition was assumed on the functions $\psi_{L}$ and $\psi_{R}$, restricting (1.1). The approach here differs from that in [3] in the realization that there is no point insisting on the deterministic centering $\int_{1}^{n} \psi_{M}(u) d u$ instead of the present $\int_{1}^{S_{n}^{(M)}} \psi_{M}(u) d u$ in $V_{n}^{(M)}$ in $(1.2), M=L, R$, and in the associated use of moment generating functions, rather than just moments, resulting in faster rates of approximation and no restriction on $L(\cdot)$ and $R(\cdot)$. As explained in [3], these approximations are made possible by a probabilistic representation of a random variable with a given, arbitrary infinitely divisible distribution, obtained in [1]. The sums $\sum_{j=1}^{n} \psi_{L}\left(S_{j}^{(L)}\right) \leqq 0$ in $V_{n}^{(L)}$ and $-\sum_{j=1}^{m} \psi_{R}\left(S_{j}^{(R)}\right) \geqq 0$ in $-V_{m}^{(R)}$ are to be viewed as the asymptotic contributions of fixed numbers, $n$ and $m$, of the smallest and the largest terms in a sum of independent and identically distributed random variables in the domain of partial attraction of the infinitely divisible law given by the quadruple $(\theta, \sigma, L(\cdot), R(\cdot))$. (For a recent discussion of such domains the reader is referred to [4].) Thus $V_{n}^{(L)}$ and $-V_{m}^{(R)}$ themselves are centered versions of these asymptotic contributions, presently with random centerings. This is why such approximations were called "extreme-sum approximations" in [3].

\section{Proof of the theorem}

On the same probability space $(\Omega, \mathcal{A}, P)$ where the random variables $V_{n, m}:=V_{n, m}(\theta, \sigma, L, R):=V_{n}^{(L)}+\sigma Z-V_{m}^{(R)}+\theta-\theta_{L}+\theta_{R}$ are defined, and expressed in terms of the same independent sequences $\left\{S_{n}^{(L)}\right\}_{n=1}^{\infty},\left\{S_{n}^{(R)}\right\}_{n=1}^{\infty}$ and $Z$, for a given quadruple $(\theta, \sigma, L, R)$ let $V:=V(\theta, \sigma, L, R):=V_{L}+\sigma Z-$ 
$V_{R}+\theta-\theta_{L}+\theta_{R}$, where for the independent left-continuous Poisson processes

$$
N_{M}(u):=\sum_{k=1}^{\infty} I\left\{S_{k}^{(M)}<u\right\}, \quad 0 \leqq u<\infty, \quad M=L, R,
$$

with unit intensity, where $I\{\cdot\}$ is the indicator function,

$V_{M}:=\int_{S_{1}^{(M)}}^{\infty}\left[u-N_{M}(u)\right] d \psi_{M}(u)+\int_{1}^{S_{1}^{(M)}} u d \psi_{M}(u)+\psi_{M}(1), \quad M=L, R$.

Then by Theorem 3 in [1], the distribution function of the variable $V=$ $=V(\theta, \sigma, L, R)$ is the function $F_{\theta, \sigma, L, R}(\cdot)$ to be estimated. Since $D_{n, m}(L, R) \leqq r_{n}^{(L)}\left(a_{L}\right)+r_{m}^{(R)}\left(a_{R}\right)$ if $P\left\{\left|V-V_{n, m}\right|>r_{n}^{(L)}\left(a_{L}\right)+r_{m}^{(R)}\left(a_{R}\right)\right\} \leqq$ $\leqq r_{n}^{(L)}\left(a_{L}\right)+r_{m}^{(R)}\left(a_{R}\right)$, the inequality claimed in the theorem will follow if we show that $P\left\{\left|V_{M}-V_{n}^{(M)}\right|>r_{n}^{(M)}(a)\right\} \leqq r_{n}^{(M)}(a)$ holds for all $n \in \mathbf{N}$ and $a \geqq$ $\geqq a_{M}^{*}$, for both $M=L$ and $M=R$. Dropping the indices in (1.4)-(1.6), i.e. setting $v^{2}(a):=\int_{a}^{\infty} \psi^{2}(u) d u$, for some $1 / 2 \leqq d \leqq 2 e^{(e-2) / 2}$ choosing $a^{*}>0$ so that

$$
\left\{\begin{array}{l}
\psi\left(a^{*}\right)=0 \text { if } \psi(a)=0 \text { for some } a>0 \text { and } \\
v\left(a^{*}\right) \leqq \frac{1}{e^{2 / e}} \text { and }\left|\psi\left(a^{*}\right)\right| \leqq \frac{1}{e^{e^{e / 2} / d}} \text { if } \psi(a)<0 \text { for all } a>0,
\end{array}\right.
$$

and for $a \geqq a^{*}$, with the same convention that $w(a)=0$ if $\psi(a)=0$, defining

$$
w(a):= \begin{cases}w_{1}(a):=\sqrt{\frac{e}{2}} v(a) \sqrt{\log \frac{1}{v(a)}}, & \text { if } \sqrt{\frac{2}{e} \log \frac{1}{v(a)}} \leqq \frac{v(a)}{|\psi(a)|}, \\ w_{2}(a):=\frac{1}{2}|\psi(a)| \log \frac{1}{v(a)|\psi(a)|}, & \text { if } \sqrt{\frac{2}{e} \log \frac{1}{v(a)}}>\frac{v(a)}{|\psi(a)|} \geqq 1, \\ w_{3}(a):=d|\psi(a)| \log \frac{1}{|\psi(a)|}, & \text { if } \sqrt{\frac{2}{e} \log \frac{1}{v(a)}}>1>\frac{v(a)}{|\psi(a)|},\end{cases}
$$

for a non-decreasing, non-positive, right-continuous function $\psi(\cdot)$ on $(0, \infty)$, for which $v(a)<\infty$ for all $a>0$, we have to show that for all $n \in \mathbf{N}$ and $a \geqq a^{*}$,

$$
P\left\{\left|\Delta_{n}\right|>r_{n}(a)\right\} \leqq r_{n}(a),
$$

where

$$
r_{n}(a):= \begin{cases}P\left\{S_{n} \leqq a\right\}+2 w(a), & \text { if } \psi(\cdot) \not \equiv 0 \\ 0, & \text { if } \psi(\cdot) \equiv 0\end{cases}
$$


and, using that the jump-points of the Poisson process $N(u):=$ $:=\sum_{k=1}^{\infty} I\left\{S_{k}<u\right\}, 0 \leqq u<\infty$, hit the possible discontinuity points of $\psi(\cdot)$ with probability zero,

$$
\begin{gathered}
\Delta_{n}:=\int_{S_{1}}^{\infty}[u-N(u)] d \psi(u)+\int_{1}^{S_{1}} u d \psi(u)+ \\
+\psi(1)-\sum_{j=1}^{n} \psi\left(S_{j}\right)+\int_{1}^{S_{n}} \psi(u) d u= \\
=\int_{S_{n}}^{\infty}[u-N(u)] d \psi(u)+\sum_{j=1}^{n-1}\left\{\int_{S_{j}}^{S_{j+1}} u d \psi(u)-j\left[\psi\left(S_{j+1}\right)-\psi\left(S_{j}\right)\right]\right\}+ \\
+\int_{1}^{S_{1}} u d \psi(u)+\psi(1)-\sum_{j=1}^{n} \psi\left(S_{j}\right)+\int_{1}^{S_{n}} \psi(u) d u= \\
=\int_{S_{n}}^{\infty}[u-N(u)] d \psi(u)+\int_{1}^{S_{n}} u d \psi(u)+\int_{1}^{S_{n}} \psi(u) d u-n \psi\left(S_{n}\right)+\psi(1)= \\
=\int_{S_{n}}^{\infty}[u-N(u)] d \psi(u)+\psi\left(S_{n}\right)\left[S_{n}-n\right]
\end{gathered}
$$

almost surely. (Throughout the usual convention $\int_{c}^{d} \cdots d \psi:=\int_{(c, d]} \cdots d \psi$ applies for all $0<c<d<\infty$. The integral on the half-line $\left(S_{1}, \infty\right)$ exists almost surely as an improper Riemann integral by (1.1), i.e. by the fact that $v(a)<\infty$ for all $a>0$.)

If $\psi(u)=0$ for all $u>0$, there is in fact nothing to prove. (And here we have $P\left\{\left|\Delta_{n}\right|>0\right\}=0$ since $\Delta_{n} \equiv 0$.) If $\psi(\cdot) \not \equiv 0$ on $(0, \infty)$, two cases are distinguished. The trivial case is when $\psi\left(a^{*}\right)=0$ and hence $\psi(v)=0$ for all $v \geqq a^{*}$. In this case, $P\left\{\left|\Delta_{n}\right|>r_{n}(a)\right\} \leqq P\left\{S_{n} \leqq a\right\}+P\left\{\left|\Delta_{n}\right|>r_{n}(a), S_{n}>\right.$ $>a\}=P\left\{S_{n} \leqq a\right\}$ and $w(a)=0$ for all $a \geqq a^{*}$, and so (2.3) follows with $r_{n}(a)=P\left\{S_{n} \leqq a\right\}$.

For the non-trivial case, suppose that $\psi(v)<0$ for all $v>0$. Fix $n \in$ $\in \mathbf{N}$ and $a \geqq a^{*}$, and put $g_{n}(x):=x^{n-1} e^{-x} /(n-1) !, x>0$, for the density function of $\bar{S}_{n}$. By the definition of $r_{n}(a)$ in (2.3) and by Markov's inequality we have

$$
\begin{gathered}
\text { (2.4) } P\left\{\left|\Delta_{n}\right|>r_{n}(a)\right\} \leqq P\left\{S_{n} \leqq a\right\}+P\left\{\Delta_{n} \geqq 2 w(a), S_{n}>a\right\}+ \\
+P\left\{-\Delta_{n} \geqq 2 w(a), S_{n}>a\right\} \leqq \\
\leqq
\end{gathered}
$$




$$
\begin{aligned}
=P\left\{S_{n} \leqq a\right\}+e^{-2 s w(a)} \int_{a}^{\infty} \exp \left\{\int_{x}^{\infty}\left[e^{s \psi(v)}-1-s \psi(v)\right] d v\right\} g_{n}(x) d x+ \\
+e^{-2 t w(a)} \int_{a}^{\infty} \exp \left\{\int_{x}^{\infty}\left[e^{-t \psi(v)}-1+t \psi(v)\right] d v\right\} g_{n}(x) d x
\end{aligned}
$$

for every $s>0$ and every $t>0$, where the last equation for the restricted moment generating functions follows by a slight modification of the first part of the proof of Theorem 4 in [1]. Actually, the slight modification is just the trivial one to account for the restrictive presence of the indicators. Indeed, that taken for granted and setting

$$
\begin{aligned}
\Delta_{n}^{*}: & =\int_{S_{n}}^{\infty}[v-N(v)] d \psi(v)+\int_{1}^{S_{n}} v d \psi(v)+ \\
& +\int_{1}^{n} \psi(v) d v-(n-1) \psi\left(S_{n}\right)+\psi(1)
\end{aligned}
$$

Theorem 4 in [1] directly gives (replacing the it there by $u$ ) that for all $u \in \mathbf{R}$,

$$
\begin{aligned}
& E\left(e^{u \Delta_{n}^{*}} I\left\{S_{n}>a\right\}\right)=\int_{a}^{\infty} \exp \left\{\int_{x}^{\infty}\left[e^{u \psi(v)}-1-\frac{u \psi(v)}{1+\psi^{2}(v)}\right] d v+u \psi(v)+\right. \\
& \left.+u \int_{1+x}^{n} \psi(v) d v+u \int_{x}^{1+x} \frac{\psi(v)}{1+\psi^{2}(v)} d v-u \int_{1+x}^{\infty} \frac{\psi^{3}(v)}{1+\psi^{2}(v)} d v\right\} g_{n}(x) d x= \\
& =\int_{a}^{\infty} \exp \left\{\int_{x}^{\infty}\left[e^{u \psi(v)}-1-u \psi(v)\right] d v+u \psi(x)+u \int_{x}^{n} \psi(v) d v\right\} g_{n}(x) d x
\end{aligned}
$$

where the second equation is by straightforward algebra. Hence for

$$
\begin{gathered}
\Delta_{n}^{*}-\psi\left(S_{n}\right)-\int_{S_{n}}^{n} \psi(v) d v=\int_{S_{n}}^{\infty}[v-N(v)] d \psi(v)+\int_{1}^{S_{n}} v d \psi(v)+ \\
\quad+\int_{1}^{S_{n}} \psi(v) d v-n \psi\left(S_{n}\right)+\psi(1)= \\
=\int_{S_{n}}^{\infty}[v-N(v)] d \psi(v)+S_{n} \psi\left(S_{n}\right)-n \psi\left(S_{n}\right)=\Delta_{n}
\end{gathered}
$$

we clearly obtain

$$
\begin{gathered}
E\left(e^{u \Delta_{n}} I\left\{S_{n}>a\right\}\right)=\int_{a}^{\infty} \exp \left\{\int_{x}^{\infty}\left[e^{u \psi(v)}-1-u \psi(v)\right] d v\right\} g_{n}(x) d x \\
u \in \mathbf{R}
\end{gathered}
$$


proving (2.4), where the integrals on the right may or may not be finite at this stage.

To estimate the integrands there, we use the inequality that if $c \geqq 0$ is a constant, then $e^{u}-1-u \leqq e^{c} u^{2} / 2$ for all $-\infty<u \leqq c$. For the first integral in (2.4), we have $-\infty<s \psi(v)<0$ for all $v \geqq a$, so that

$$
e^{s \psi(v)}-1-s \psi(v) \leqq \frac{s^{2}}{2} \psi^{2}(v), \quad v \geqq a, \quad \text { for every } \quad s>0 .
$$

For the second, since the negative function $\psi(\cdot)$ is non-decreasing, we obviously have $0<-t \psi(v)=t|\psi(v)| \leqq|\psi(v)| /|\psi(a)| \leqq 1$ whenever $0<t \leqq$ $\leqq 1 /|\psi(a)|$ and $v \geqq a$, so that

$$
e^{-t \psi(v)}-1+t \psi(v) \leqq \frac{e t^{2}}{2} \psi^{2}(v), \quad v \geqq a, \quad \text { for every } \quad 0<t \leqq \frac{1}{|\psi(a)|} .
$$

Hence, moving down $x$ to $a$ in the integrals in both exponents, from (2.4) we obtain

$$
\begin{gathered}
P\left\{\left|\Delta_{n}\right|>r_{n}(a)\right\} \leqq \\
\leqq P\left\{S_{n} \leqq a\right\}+\exp \left\{\frac{s^{2}}{2} v^{2}(a)-2 s w(a)\right\}+\exp \left\{\frac{e t^{2}}{2} v^{2}(a)-2 t w(a)\right\}
\end{gathered}
$$

for all $s>0$ and $0<t \leqq 1 /|\psi(a)|$.

Using (2.2), for all choices of $a \geqq a^{*}$ for which $w(a)=w_{2}(a)$ we have

$$
w_{2}(a)>\frac{1}{2} \sqrt{\frac{e}{2}} \frac{v(a)}{\sqrt{\log \frac{1}{v(a)}}} \log \frac{1}{v^{2}(a)}=\sqrt{\frac{e}{2}} v(a) \sqrt{\log \frac{1}{v(a)}}=w_{1}(a) .
$$

Also, for all $a \geqq a^{*}$ for which $w(a)=w_{3}(a)$ the choice of $a^{*}$ in (2.1) forces

$$
\begin{gathered}
x:=\frac{1}{v(a)}>\frac{1}{|\psi(a)|} \geqq e^{e^{e / 2} / d}>e^{e / d} \geqq e^{\frac{e}{2 d^{2}}} \text { since } \\
v(a)<|\psi(a)| \text { and } \cdot d \geqq \frac{1}{2} .
\end{gathered}
$$

This implies that $\sqrt{\log x}>\sqrt{e / 2} / d$ or, what is of course the same, $\sqrt{e / 2} /$ $/(d \sqrt{\log x})<1$ and, consequently, $\sqrt{e / 2} x /(d \log x)<x / \sqrt{\log x}$. So,

$$
\frac{1}{d} \sqrt{\frac{e}{2}} \frac{y}{\log y}<\frac{1}{d} \sqrt{\frac{\epsilon}{2}} \frac{x}{\log x} \leqq \frac{x}{\sqrt{\log x}}
$$


whenever $y:=1 /|\psi(a)|<x$, since the function $y / \log y, y>0$, is increasing on the half-line $[e, \infty)$ and $y=1 /|\psi(a)|>e$ by the choice of $a^{*}$ and the upper bound on $d$. But by (2.2) the inequality $y=1 /|\psi(a)|<1 / v(a)=$ $=x$ is equivalent to $w(a)=w_{3}(a)$. Thus, if $a \geqq a^{*}$ and $w(a)=w_{3}(a)$, then $\sqrt{e / 2} y / \log y<d x / \sqrt{\log x}$ or, what is the same,

$$
\sqrt{\frac{e}{2}} \frac{1}{|\psi(a)| \log \frac{1}{|\psi(a)|}}<d \frac{1}{v(a) \sqrt{\log \frac{1}{v(a)}}}, \quad \text { that is, } w_{1}(a)<w_{3}(a) .
$$

(We see that $w_{2}(a)>w_{1}(a)$ whenever $v(a)<1,|\psi(a)|<1$ and $w(a)=$ $=w_{2}(a)$.) For reference purposes the foregoing may be summarized by saying that whenever $a \geqq a^{*}$,

$$
\text { if } w(a)=w_{j}(a), \text { then } w_{j}(a)>w_{1}(a), \quad j=2,3 .
$$

Consider the convex function $f_{a}(s):=\frac{s^{2}}{2} v^{2}(a)-2 s w(a), s>0$. Then $f_{a}(\cdot)$ is negative on the interval $\left(0,4 w(a) / v^{2}(a)\right)$ and takes its minimum at $s_{*}=2 w(a) / v^{2}(a)$. Hence, choosing $s=s_{*}$ and using (2.6) twice, the second term of the bound in (2.5) is

$$
\begin{gathered}
\exp \left\{f_{a}\left(s_{*}\right)\right\}=\exp \left\{-\frac{2 w^{2}(a)}{v^{2}(a)}\right\} \leqq \exp \left\{-\frac{2 w_{1}^{2}(a)}{v^{2}(a)}\right\}=\exp \left\{-e \log \frac{1}{v(a)}\right\}< \\
<v(a) \leqq w_{1}(a) \leqq w(a) .
\end{gathered}
$$

The inequality before the last holds since $v(a) \leqq e^{-2 / e}$ for all $a \geqq a^{*}$ by (2.1).

The convex function $h_{a}(t):=\frac{e t^{2}}{2} v^{2}(a)-2 t w(a), t>0$, is also negative on the interval $\left(0,[4 w(a)] /\left[e v^{2}(a)\right]\right)$ and takes its minimum at the point $t_{*}:=$ $:=[2 w(a)] /\left[e v^{2}(a)\right]$. However, here we also have to satisfy the constraint $0<$ $<t \leqq 1 /|\psi(a)|$. So, choosing $t_{0}:=\min \left\{1 /|\psi(a)|, t_{*}\right\}$, the third term of the bound in (2.5) becomes $\exp \left\{h_{a}\left(t_{\diamond}\right)\right\}$. Let $a \geqq a^{*}$. If $w(a)=w_{1}(a)$, so that

$$
0<t_{*}=\frac{2 w_{1}(a)}{e v^{2}(a)}=\sqrt{\frac{2}{e}} \frac{\sqrt{\log \frac{1}{v(a)}}}{v(a)} \leqq \frac{1}{|\psi(a)|},
$$

we have (whenever $v(a) \leqq e^{-2 / e}$ as above)

$$
\begin{gathered}
\exp \left\{h_{a}\left(t_{\diamond}\right)\right\}=\exp \left\{h_{a}\left(t_{*}\right)\right\}=\exp \left\{\log \frac{1}{v(a)}-2 t_{*} w_{1}(a)\right\}= \\
=\exp \left\{\log \frac{1}{v(a)}-2 \log \frac{1}{v(a)}\right\}=v(a) \leqq w_{1}(a)=w(a) .
\end{gathered}
$$


If $w(a)=w_{j}(a), j=2,3$, then by $(2.6)$ again,

$$
t_{*}=\frac{2 w_{j}(a)}{e v^{2}(a)}>\frac{2 w_{1}(a)}{e v^{2}(a)}=\sqrt{\frac{2}{e}} \frac{\sqrt{\log \frac{1}{v(a)}}}{v(a)}>\frac{1}{|\psi(a)|}, \quad j=2,3 .
$$

Hence if $w(a)=w_{2}(a)$, then (whenever $v(a)<1,|\psi(a)|<1$ and $v(a)|\psi(a)| \leqq$ $\left.\leqq e^{-2}\right)$

$$
\begin{gathered}
\exp \left\{h_{a}\left(t_{\diamond}\right)\right\}=\exp \left\{h_{a}(1 /|\psi(a)|)\right\}= \\
=\exp \left\{\frac{e}{2} \frac{v^{2}(a)}{\psi^{2}(a)}-\frac{2 w_{2}(a)}{|\psi(a)|}\right\}<\frac{1}{v(a)} \exp \left\{-\frac{2 w_{2}(a)}{|\psi(a)|}\right\}= \\
=\frac{1}{v(a)} \exp \left\{-\log \frac{1}{v(a)|\psi(a)|}\right\}=|\psi(a)| \leqq w_{2}(a)=w(a)
\end{gathered}
$$

by the choice of $a^{*}$, while if $w(a)=w_{3}(a)$, then

$$
\begin{gathered}
\exp \left\{h_{a}\left(t_{\diamond}\right)\right\}=\exp \left\{h_{a}(1 /|\psi(a)|)\right\}= \\
=\exp \left\{\frac{e}{2} \frac{v^{2}(a)}{\psi^{2}(a)}-\frac{2 w_{3}(a)}{|\psi(a)|}\right\}<e^{e / 2} \exp \left\{-\frac{2 w_{3}(a)}{|\psi(a)|}\right\}= \\
=e^{e / 2}|\psi(a)|^{2 d} \leqq e^{e / 2}|\psi(a)| \leqq d|\psi(a)| \log \frac{1}{|\psi(a)|}=w_{3}(a)=w(a)
\end{gathered}
$$

since $2 d \geqq 1$ and $|\psi(a)| \leqq 1 / e^{e / 2} / d$ by the choice of $a^{*}$. Therefore, the inequality $\exp \left\{h_{a}\left(t_{\diamond}\right)\right\}<w(a)$ holds for all $a \geqq a^{*}$.

Thus if $a \geqq a^{*}$, then the bound in (2.5) is less than $P\left\{S_{n} \leqq a\right\}+2 w(a)=$ $=r_{n}(a)$. This fact establishes (2.3) in the non-trivial case, and hence the theorem. (The collection of bracketed phrases also establishes the remark concerning the choice of the thresholds.)

\section{Examples}

The first three examples show, in particular, that all three versions of the rate function provided by the three branches of $w_{M}(\cdot), M=L, R$, defined between (1.5) and (1.6), may in fact occur. For simplicity of exposition, we deal with spectrally one-sided infinitely divisible distributions, that is, we choose $L(\cdot) \equiv 0$, with the exception of the stable and compound Poisson examples. The last four examples are of interest in their own right, the negative binomial being weird enough to deserve attention in any case. In Examples 2-4, the threshold remark beneath the theorem is used without further notice. 
EXAMPLE 1. If $L \equiv 0$ and $\psi_{R}(u)=-e^{-u}, u>0$, then $w_{L}(a)=0$, $v_{R}(a)=e^{-a} / \sqrt{2}$ and $w_{R}(a)=w_{3}^{(R)}(a)=d a e^{-a}$ for all $a>0$ for $v_{R}(a) /$ $/\left|\psi_{R}(a)\right|=1 / \sqrt{2}<1$. Regardless of $\theta \in \mathbf{R}$ and $\sigma \geqq \mathbf{0}$, for the corresponding Lévy distance the theorem and (1.8), with $a_{n} \equiv(2-\sqrt{3}) n$, give

$$
D_{n, n}(0, R) \leqq \frac{3 d(2-\sqrt{3}) n}{\exp \{(2-\sqrt{3}) n\}} \quad \text { for all } \quad n \geqq \frac{\max \left(\frac{\mathrm{e}^{e / 2}}{d}, \frac{2}{\epsilon}-\log \sqrt{2}\right)}{2-\sqrt{3}}
$$

and each fixed $1 / 2 \leqq d \leqq 2 e^{(e-2) / 2}=2.86419 \ldots$ For $d=2 e^{(e-2) / 2}$ this holds for all $n \geqq 6$ and for $d=1 / 2$ the inequality is true for all $n \geqq 30$.

EXAMPLE 2 . If $L \equiv 0$ and $\psi_{R}(u)=-\sqrt{u} e^{-u / 2}$, then $w_{L}(a)=0, v_{R}(a)=$ $=\sqrt{a+1} e^{-a / 2}$ and $w_{R}(a)=w_{2}^{(R)}(a)=2^{-1} \sqrt{a} e^{-a / 2} \log \left(e^{a}\left[a^{2}+a\right]^{-1 / 2}\right)$ for all $a \geqq 2 /(e-2)=2.78442 \ldots$, say. Regardless of $\theta \in \mathbf{R}$ and $\sigma \geqq 0$, for the corresponding Lévy distance the theorem and (1.8), with $a_{n} \equiv(3-\sqrt{5}) n / 2$, give

$$
\begin{aligned}
& D_{n, n}(0, R) \leqq \frac{3 \sqrt{(3-\sqrt{5}) n}}{2^{3 / 2} \exp \left\{\frac{3-\sqrt{5}}{4} n\right\}} \log \frac{\exp \left\{\frac{3-\sqrt{5}}{2} n\right\}}{\sqrt{\frac{3-\sqrt{5}}{2} n} \sqrt{1+\frac{3-\sqrt{5}}{2} n}} \\
& \quad \text { for all } n \geqq 5>\frac{3.35}{3-\sqrt{5}} .
\end{aligned}
$$

EXAMPLE 3: Stable laws. Let $F_{\alpha, \beta, \eta, \zeta}(\cdot)$ be the distribution function of a non-normal stable law with exponent $0<\alpha<2$, given by its characteristic function

$$
\begin{gathered}
\int_{-\infty}^{\infty} e^{i t x} d F_{\alpha, \beta, \eta, \zeta}(x)= \\
= \begin{cases}\exp \left\{i \zeta t-\eta|t|^{\alpha}[1-i \beta \operatorname{sgn}(t) \tan (\alpha \pi / 2)]\right\}, & \text { if } \alpha \neq 1, \\
\exp \left\{i \zeta t-\eta|t|^{\alpha}\left[1+i \beta \operatorname{sgn}(t) \frac{2}{\pi} \log |t|\right]\right\}, & \text { if } \alpha=1,\end{cases}
\end{gathered}
$$

with skewness, scale and location parameters $-1 \leqq \beta \leqq 1, \eta>0$ and $\zeta \in \mathbf{R}$, where $\operatorname{sgn}(t)$ is the sign function, $t \in \mathbf{R}$. In Lévy's canonical form at the beginning of the paper, this is given by some $\theta=\theta(\alpha, \beta, \eta, \zeta), \sigma=0$ and $L(\cdot)$ and $R(\cdot)$ functions such that $\psi_{M}(u)=-c_{M} u^{-1 / \alpha}, u>0$, where $c_{M}=$ $=c_{M}(\alpha, \beta, \eta, \zeta) \geqq 0$ are some constants, $M=L, R$, such that $c_{L}(\alpha, 1, \eta, \zeta)=$ $=0$ and $c_{L}(\alpha, \beta, \eta, \zeta)>0$ for every $-1 \leqq \beta<1$, while $c_{R}(\alpha,-1, \eta, \zeta)=0$ and $c_{R}(\alpha, \beta, \eta, \zeta)>0$ for every $-1<\beta \leqq 1 ;$ cf. [9], [1], [4]. Setting $K_{\alpha, \beta, \eta, \zeta}:=$ $:=\sup \left\{f_{\alpha, \beta, \eta, \zeta}(x): x \in \mathbf{R}\right\}<\infty$ for the corresponding density function 
$f_{\alpha, \beta, \eta, \zeta}(\cdot):=F_{\alpha, \beta, \eta, \zeta}^{\prime}(\cdot)$ and $\vartheta=\vartheta(\alpha, \beta, \eta, \zeta):=\theta-\theta_{L}+\theta_{R}$, where $\theta_{L}$ and $\theta_{R}$ are given through (1.3), let $F_{\alpha, \beta, \eta, \zeta}^{n, m}(x):=P\left\{V_{n}^{(L)}-V_{m}^{(R)}+\vartheta \leqq x\right\}, x \in \mathbf{R}$, where, in the present situation $V_{n}^{(M)}$ of $(1.2)$, for $M=L, R$ and $n \in \mathrm{N}$, is given by

$$
V_{n}^{(M)}= \begin{cases}-c_{M} \sum_{j=1}^{n}\left(S_{j}^{(M)}\right)^{-1 / \alpha}+\frac{\alpha c_{M}}{\alpha-1}\left(S_{n}^{(M)}\right)^{\frac{\alpha-1}{\alpha}}-\frac{\alpha c_{M}}{\alpha-1}, & \text { if } \alpha \neq 1, \\ -c_{M} \sum_{j=1}^{n}\left(S_{j}^{(M)}\right)^{-1}+c_{M} \log S_{n}^{(M)}, & \text { if } \alpha=1 .\end{cases}
$$

Elementary calculation shows that $v_{M}(a)=\sqrt{\alpha /(2-\alpha)} c_{M} a^{-(2-\alpha) /(2 \alpha)}, a>$ $>0$, and

$$
\begin{gathered}
w_{M}(a)=w_{1}^{(M)}(a)=\frac{\sqrt{e}}{2} \frac{c_{M}}{a^{\frac{1}{\alpha}-\frac{1}{2}}} \sqrt{\log \left(a\left[c_{M} \sqrt{\alpha /(2-\alpha)}\right]^{-2 \alpha /(2-\alpha)}\right)} \\
\text { for all } a \geqq a_{M}^{*}
\end{gathered}
$$

if $c_{M}>0$, where, putting $\rho:=\alpha /(2-\alpha), u_{M}:=(2 /(\rho e)) \log \left(1 /\left(c_{M} \sqrt{\rho}\right)\right)$ and $v_{M}:=1 /\left(2 \rho^{2}\right)$, the threshold $a_{M}^{*}$ may be chosen as $a_{M}^{*}=$ $=\max \left(\rho^{\rho} c_{M}^{2 \rho} e^{4 \rho / e}, a_{M}^{\diamond}\right)$, where $a_{M}^{\diamond}$ is the smallest positive number such that $a \geqq u_{M}+v_{M} \log a$ for all $a \geqq a_{M}^{\circ}, M=L, R$. Picking now any $\tau \in(0,1)$ in (1.8) and letting $n_{M}^{*}:=\max \left(a_{M}^{*} / \tau, n_{M}^{\diamond}\right)$, where $n_{M}^{\diamond}$ is the smallest $n \in \mathbf{N}$ for which $\exp \left\{-(1-\tau)^{2} n / 2\right\} \leqq w_{1}^{(M)}(\tau n), M=L, R$, the inequality in (1.9) gives that for all $n \geqq n_{L}^{*}$ and $m \geqq n_{R}^{*}$,

$$
\sup _{x \in \mathbf{R}}\left|F_{\alpha, \beta, \eta, \zeta}^{n, m}(x)-F_{\alpha, \beta, \eta, \zeta}(x)\right| \leqq 3\left[1+K_{\alpha, \beta, \eta, \zeta}\right]\left[w_{1}^{(L)}(\tau n)+w_{1}^{(R)}(\tau m)\right] .
$$

Neglecting thresholds and constants, the qualitative meaning of this is that

$$
\begin{gathered}
\sup _{x \in \mathbf{R}}\left|F_{\alpha, \beta, \eta, \zeta}^{n, m}(x)-F_{\alpha, \beta, \eta, \zeta}(x)\right|=O\left(c_{L} \frac{\sqrt{\log n}}{n^{\frac{1}{\alpha}-\frac{1}{2}}}+c_{R} \frac{\sqrt{\log m}}{m^{\frac{1}{\alpha}-\frac{1}{2}}}\right) \\
\text { as } n, m \rightarrow \infty .
\end{gathered}
$$

Improving Theorem 2.2 in [2], the latter rate has also been established in Remark 1.3 of Janssen and Mason [11] by completely different methods.

Example 4: Limiting St. Petersburg distributions. In a classical St. Petersburg game, a player gains $2^{k}$ ducats with probability $2^{-k}, k \in \mathbf{N}$. As determined in [5], the class $\left\{G_{\gamma}(\cdot): 1 / 2<\gamma \leqq 1\right\}$ of all possible nondegenerate subsequential limiting types of distribution functions for the cumulative gains of a player in a sequence of independent St. Petersburg games, 
under any deterministic centering and norming, is described by the family of infinitely divisible characteristic functions

$$
\int_{-\infty}^{\infty} e^{i t x} d G_{\gamma}(x)=\exp \left\{i \theta_{\gamma} t+\int_{0}^{\infty}\left(e^{i t x}-1-\frac{i t x}{1+x^{2}}\right) d R_{\gamma}(x)\right\}, \quad t \in \mathbf{R}
$$

where, with Log standing for the logarithm to the base 2 and, for any $y \in \mathbf{R}$, with $\lfloor y\rfloor$ denoting the greatest integer not greater than $y$, having a fractional part $\langle y\rangle=y-\lfloor y\rfloor$,

$$
\theta_{\gamma}:=\sum_{k=1}^{\infty} \frac{\gamma^{2}}{\gamma^{2}+4^{k}}-\sum_{k=0}^{\infty} \frac{1}{1+\gamma^{2} 4^{k}}-\log \gamma
$$

and

$$
R_{\gamma}(x)=-\gamma 2^{-\lfloor\log (\gamma x)\rfloor}, \quad x>0,
$$

so that $\psi_{\gamma}(u):=\psi_{R_{\gamma}}(u)=-2^{-\lfloor\log (u / \gamma)\rfloor} / \gamma, u>0$. Hence by lengthier but elementary and quite delicate computation through (1.2) and (1.3),

$$
W_{n}^{(\gamma)}:=-V_{n}^{\left(R_{\gamma}\right)}+\theta_{\gamma}+\theta_{R_{\gamma}}=\frac{1}{\gamma} \sum_{j=1}^{n} \frac{1}{2^{\left\lfloor\log \left(S_{j} / \gamma\right)\right\rfloor}}-\log S_{n}+\delta\left(\frac{S_{n}}{\gamma}\right),
$$

where $\delta(s)=1+\langle\log s\rangle-2^{\langle\log s\rangle}, s>0$, and it can be seen in similarly elementary fashion that $0 \leqq \delta(s) \leqq 1-(1+\log \log 2) / \log 2=0.08607 \ldots$ for all $s>0$. (The function $\delta(\cdot)$ plays a special role in the theory of the St. Petersburg game, described in [6], and the present example has some motivational value at some point there.) Also, since $1 / u \leqq\left|\psi_{\gamma}(u)\right|<2 / u$ for all $u>0$, for the corresponding $v_{\gamma}^{2}(a):=\int_{a}^{\infty} \psi_{\gamma}^{2}(u) d u$ we obtain $1 / \sqrt{a} \leqq v_{\gamma}(a)<\sqrt{2} / \sqrt{a}$ for every $a>0$ and $1 / 2<\gamma \leqq 1$. Thus we have $v_{\gamma}(a)<e^{-2 / e}$ if $a \geqq a^{*}:=$ $:=2 e^{4 / e}=8.71168 \ldots$ and $\sqrt{a} / 2<v_{\gamma}(a) /\left|\psi_{\gamma}(u)\right| \leqq \sqrt{2 a}$, so

$$
w_{R_{\gamma}}(a)=w_{1}^{\left(R_{\gamma}\right)}(a)=\sqrt{\frac{e}{2}} v_{\gamma}(a) \sqrt{\log \left[1 / v_{\gamma}(a)\right]}<\sqrt{\frac{e}{2}} \frac{\sqrt{\log a}}{\sqrt{a}}=: w_{1}(a)
$$

for all $a>1$ and all $1 / 2<\gamma \leqq 1$. Since the densities $g_{\gamma}(\cdot)=G_{\gamma}^{\prime}(\cdot)$ exist and it can be shown that $\sup _{1 / 2<\gamma \leqq 1} \sup \left\{g_{\gamma}(x): x \in \mathbf{R}\right\} \leqq 1 / 2$, for any $0<\tau<1$ in (1.8), finally (1.9) yields

$$
\sup _{\frac{1}{2}<\gamma \leqq 1} \sup _{x \in \mathbf{R}}\left|P\left\{W_{n}^{(\gamma)} \leqq x\right\}-G_{\gamma}(x)\right|<C(\tau) \frac{\sqrt{\log n}}{\sqrt{n}} \text { for all } n \geqq n^{*}(\tau),
$$


where the bound is a trivial upper bound for $3 w_{1}(\tau n)$ with $C(\tau):=9 \sqrt{e /(2 \tau)} / 2$ and $n^{*}(\tau):=\left\lceil\max \left(2 e^{4 / e} / \tau, n_{*}(\tau)\right)\right\rceil$, where $\lceil x\rceil:=\min \{k \in \mathbf{N}: k \geqq x\}, \quad x>0, \quad$ and $\quad n_{*}(\tau):=\min \{k \in \mathbf{N}:$ $\left.: \exp \left\{-(1-\tau)^{2} k\right\} \leqq w_{1}^{2}(\tau k)\right\}$. Here, rounding up, $C(1)=5.24620$ is unachievable, and we get $C(0.707)=6.23929, n^{*}(0.707)=13 ; \quad C(0.8)=$ $=5.86543, \quad n^{*}(0.8)=53 ; \quad C(0.9)=5.52998, \quad n^{*}(0.9)=376 ; \quad C(0.95)=$ $=5.38249, \quad n^{*}(0.95)=2107 ; \quad C(0.99)=5.27263, \quad n^{*}(0.99)=86177$ and $C(0.999)=5.24883, n^{*}(0.999)=13297850$.

Example 5: Compound Paisson laws. Let $N_{\lambda}, X_{1}, X_{2}, \ldots$ be independent random variables such that $N_{\lambda}$ has the Poisson distribution on the integers $\{0,1,2, \ldots\}$ with mean $\lambda>0$ and $X_{1}, X_{2}, \ldots$ have the same distribution function $G(x):=P\{X \leqq x\}, x \in \mathbf{R}$. Then Lévy's canonical form of the characteristic function of the infinitely divisible compound Poisson distribution function $F_{\lambda, G}(x):=P\left\{\sum_{k=1}^{N_{\lambda}} X_{k} \leqq x\right\}, x \in \mathbf{R}$, is given by $\sigma=0$ and, with $G_{-}(\cdot)$ denoting the left-continuous version of $G(\cdot)$,

$$
\begin{gathered}
\theta=\theta_{\lambda, G}=\lambda \int_{-\infty}^{\infty} \frac{x}{1+x^{2}} d G(x), \quad L(x)=\lambda G_{-}(x), x<0, \\
R(x)=\lambda[G(x)-1], x>0 .
\end{gathered}
$$

Hence, letting $G_{+}^{-1}(\cdot)$ denote the right-continuous version of the left-continuous generalized inverse $G^{-1}(s):=\inf \{x \in \mathbf{R}: G(x) \geqq s\}, 0<s<1$, the usual quantile function, pertaining to $G(\cdot)$, we have

$$
\psi_{L}(u)= \begin{cases}G_{+}^{-1}\left(\frac{u}{\lambda}\right), & \text { if } 0<u<\lambda G_{-}(0), \\ 0, & \text { if } u \geqq \lambda G_{-}(0),\end{cases}
$$

and

$$
\psi_{R}(u)= \begin{cases}-G^{-1}\left(1-\frac{u}{\lambda}\right), & \text { if } 0<u<\lambda[1-G(0)], \\ 0, & \text { if } u \geqq \lambda[1-G(0)] .\end{cases}
$$

For the Lévy distance $D_{n, m}(\lambda, G)$ between $F_{\lambda, G}(\cdot)$ and its approximation $F_{\lambda, G}^{n, m}(x):=P\left\{V_{n}^{(L)}-V_{m}^{(R)}+\theta_{\lambda, G}-\theta_{L}+\theta_{R} \leqq x\right\}, x \in \mathbf{R}$, given by the present $\psi_{L}(\cdot)$ and $\psi_{R}(\cdot)$ through (1.2) and (1.3), by (1.10) we obtain

$$
D_{n, m}(\lambda, G) \leqq \frac{\left[\lambda G_{-}(0)\right]^{n}}{n !}+\frac{[\lambda\{1-G(0)\}]^{m}}{m !} \text { for all } n, m \in \mathbf{N} .
$$

The Poisson law itself, with mean $\lambda$, is the special case when $G(x), x \in \mathbf{R}$, degenerates at the point $x=1$ and $\theta_{\lambda}:=\theta_{\lambda, G}=\lambda / 2$ for the corresponding 
quantity. In this case, $\psi_{L}(u)=0$ and $\psi_{R}(u)=-I\{u<\lambda\}$ for all $u>0$, and enjoyable calculation shows that $-V_{n}^{(R)}+\theta_{\lambda}+\theta_{R}=\sum_{j=1}^{n} I\left\{S_{j}<\lambda\right\}+$ $+\left(\lambda-S_{n}\right) I\left\{S_{n}<\lambda\right\}$ for every $n \in \mathbf{N}$. If $D_{n}(\lambda)$ denotes the Lévy distance between the distribution function $F_{n, \lambda}(\cdot)$ of the latter random variable and the Poisson distribution function $F_{\lambda}(x):=e^{-\lambda} \sum_{k=1}^{\lfloor x\rfloor} \lambda^{k} / k !, x \in \mathbf{R}$, of $N_{\lambda}$, with an empty sum understood as zero as above, then the result reduces to the inequality $D_{n}(\lambda) \leqq \lambda^{n} / n$ ! for all $n \in \mathbf{N}$. Furthermore, if $D_{n}^{*}(\lambda)$ is the Lévy distance between $F_{\lambda}(\cdot)$ and $F_{n, \lambda}^{*}(x):=P\left\{\sum_{j=1}^{n} I\left\{S_{j}<\lambda\right\} \leqq x\right\}, x \in$ $\in \mathbf{R}$, then a trivial extra step based on the triangle inequality for a Lévy distance yields $D_{n}^{*}(\lambda) \leqq 2 \lambda^{n} / n$ ! for all $n \in \mathbf{N}$.

Example 6: Negative binomial distributions. For a fixed order $\ell \in \mathbf{N}$ and success probability $0<p<1$, consider the negative binomial distribution function

$$
F_{\ell, p}(x):=P\left\{V_{\ell}(p) \leqq x\right\}:=p^{\ell} \sum_{k=\ell}^{\lfloor x\rfloor}\left(\begin{array}{c}
k-1 \\
\ell-1
\end{array}\right) q^{k-\ell}, \quad x \in \mathbf{R},
$$

where $q:=1-p$. As is well known, it is infinitely divisible and it is a routine exercise to show that the Lévy form of the characteristic function is

$$
\int_{-\infty}^{\infty} e^{i t x} d F_{\ell, p}(x)=\exp \left\{i \theta_{\ell, p} t+\int_{0}^{\infty}\left(e^{i t x}-1-\frac{i t x}{1+x^{2}}\right) d R_{\ell, p}(x)\right\}, \quad t \in \mathbf{R}
$$

where

$$
\theta_{\ell, p}=\ell+\ell \sum_{m=1}^{\infty} \frac{q^{m}}{1+m^{2}} \quad \text { and } \quad R_{\ell, p}(x)=\ell \sum_{m=1}^{\lfloor x\rfloor} \frac{q^{m}}{m}+\ell \log p, \quad x>0 .
$$

So, noting that $-\ell \log p=\ell \sum_{m=1}^{h_{\infty}} q^{m} / m$,

$$
\begin{gathered}
\psi_{\ell, p}(u):=\psi_{R_{\ell, p}}(u)=-\sum_{k=1}^{\infty} k I\left\{\log \frac{1}{p^{\ell}}-\ell \sum_{m=1}^{k} \frac{q^{m}}{m} \leqq u<\log \frac{1}{p^{\ell}}-\ell \sum_{m=1}^{k-1} \frac{q^{m}}{m}\right\}, \\
u>0,
\end{gathered}
$$

thus $\psi_{\ell, p}(u)=0$ for all $u \geqq-\ell \log p$. Evaluating (1.2) and (1.3) with this, the result is

$$
W_{n}^{\ell, p}:=-V_{n}^{\left(R_{\ell, p}\right)}+\theta_{\ell, p}+\theta_{R_{\ell, p}}=
$$




$$
\begin{gathered}
=\ell+\sum_{j=1}^{n} \sum_{k=1}^{\infty} k I\left\{\ell \sum_{m=k+1}^{\infty} \frac{q^{m}}{m} \leqq S_{j}<\ell \sum_{m=k}^{\infty} \frac{q^{m}}{m}\right\}+ \\
+\sum_{k=1}^{\infty} k\left[\ell \sum_{m=k}^{\infty} \frac{q^{m}}{m}-S_{n}\right] I\left\{\ell \sum_{m=k+1}^{\infty} \frac{q^{m}}{m} \leqq S_{n}<\ell \sum_{m=k}^{\infty} \frac{q^{m}}{m}\right\}=: \ell+T_{n}^{\ell, p}+R_{n}^{\ell, p} .
\end{gathered}
$$

(Note that $P\left\{W_{n}^{\ell, p}=\ell\right\}=P\left\{\ell+T_{n}^{\ell, p}=\ell\right\}=p^{\ell}=P\left\{V_{\ell}(p)=\ell\right\}$.) If now $D_{n}(\ell, p)$ is the Lévy distance between $F_{\ell, p}(\cdot)$ and $P\left\{W_{n}^{\ell, p} \leqq \cdot\right\}$ and $D_{n}^{*}(\ell, p)$ is the Lévy distance between $F_{\ell, p}(\cdot)$ and $P\left\{\ell+T_{n}^{\ell, p} \leqq \cdot\right\}$, then (1.10) and an extra step as above yield

$$
D_{n}(\ell, p) \leqq \frac{[-\ell \log p]^{n}}{n !} \text { and } \quad D_{n}^{*}(\ell, p) \leqq 2 \frac{[-\ell \log p]^{n}}{n !} \quad \text { for all } \quad n \in \mathbf{N}
$$

If $\ell=1$, this is of course a result for the approximation of the geometric distribution function $F_{1, p}(x)=p \sum_{k=1}^{\lfloor x\rfloor} q^{k-1}, x \in \mathbf{R}$.

I thank my daughter Zsuzsi for checking the numerical calculations in this section.

\section{References}

[1] S. Csörgö, E. Haeusler and D. M. Mason, A probabilistic approach to the asymptotic distribution of sums of independent, identically distributed random variables, Adv. in Appl. Math., 9 (1988), 259-333.

[2] S. Csörgö, Notes on extreme and self-normalised sums from the domain of attraction of a stable law, J. London Math. Soc., 39 (1989), 369-384.

[3] S. Csörgö, An extreme-sum approximation to infinitely divisible distributions without a normal component, in: Probability on vector spaces $I V$ (S. Cambanis and A. Weron, eds.), pp. 47-58, Lecture Notes in Math. 1391, Springer (Berlin, 1989).

[4] S. Csörgö, A probabilistic approach to domains of partial attraction, Adv, in Appl. Math., 11 (1990), 282-327.

[5] S. Csörgő and R. Dodunekova, Limit theorems for the Petersburg game, in: Sums, trimmed sums and extremes (M.G. Hahn, D.M. Mason and D.C. Weiner, eds.), pp. 285-315, Progress in Probability 23, Birkhäuser (Boston, 1991).

[6] S. Csörgö and G. Simons, Resolution of the St. Petersburg paradox, book manuscript in progress.

[7] L. Devroye, Laws of the iterated logarithm for order statistics of uniform spacings, Ann. Probab., 11 (1981), 860-867.

[8] A. Erdélyi, W. Magnus, F. Oberhettinger and F. G. Tricomi,.Higher transcendental functions, Vol. II, McGraw-Hill (New York, 1953).

[9] B. V. Gnedenko and A. N. Kolmogorov, Limit distributions for sums of independent random variables, Addison-Wesley (Cambridge, Massachusetts, 1954). 
[10] P. Hall, On the extreme terms of a sample from the domain of attraction of a stable law, J. London Math. Soc., 18 (1978), 181-191.

[11] A. Janssen and D. M. Mason, On the rate of convergence of sums of extremes to a stable law, Probab. Theory Related Fields, 86 (1990), 253-264.

(Received March 3, 1994)

DEPARTMENT OF STATISTICS

THE UNIVERSITY OF MICHIGAN

1444 MASON HALL

ANN ARBOR, MI 48109-1027

USA 